\title{
Descrição do perfil epidemiológico: pacientes internados por acidente vascular encefálico em uma unidade de terapia intensiva
}

\author{
Description of the epidemiological profile: patients hospitalized for stroke in an intensive care \\ Descripción del perfil epidemiológico: pacientes hospitalizados por ictus en una unidad de cuidados \\ intensivos
}

Recebido: 26/01/2022 | Revisado: 30/01/2022 | Aceito: 09/02/2022 | Publicado: 14/02/2022

\author{
Karla Azevedo da Mata \\ ORCID: https://orcid.org/0000-0002-7129-5868 \\ Secretaria Municipal da Saúde de São Paulo, Brasil \\ E-mail: karlaazevedosc@gmail.com \\ Claudia S. Pagotto Cassavia \\ ORCID: https://orcid.org/0000-0002-8561-097X \\ Secretaria Municipal da Saúde de São Paulo, Brasil \\ E-mail: cspcassavia@prefeitura.sp.gov.br \\ Joel Malaquias Junior \\ ORCID: https://orcid.org/0000-0002-1614-5373 \\ Secretaria Municipal de Saúde de São Paulo, Brasil \\ E-mail: hmcc.urgencia@gmail.com
}

\begin{abstract}
Resumo
Objetivo geral: Descrever a incidência e o perfil da população acometida por Acidente Vascular Encefálico internado na Unidade de terapia intensiva adulta (UTI) de um Hospital Público da cidade de São Paulo. Métodos: Pesquisa transversal, quantitativa, analítica e prospectiva. Coleta de dados realizada por meio de questionário, informações dos prontuários e completadas pelo paciente ou responsável. A mostra selecionada por conveniência, composta por 21 indivíduos internados na UTI com diagnóstico de AVE, entre os meses de Agosto a Outubro de 2021. Resultados: Internaram 261 pacientes, sendo $21(8,17 \%)$ com diagnóstico de AVE; 14 (66,67\%) com AVEI, sexo masculino (57,14\%), 7 (33\%) com AVEH, sexo feminino (57,14\%), os dois foram prevalentes em pessoas com mais de 60 anos (76\%), da raça branca $(61 \%) .61 \%$ dos pacientes com acompanhamento prévio em saúde, prevalente no serviço público de saúde (85\%). A maior procura do serviço de saúde prévia a internação foi o AVEI $(64,28 \%)$. Entre os AVE, 18 tinham comorbidades (HAS 66,67\%, tabagismo 42,46\%, DM 33,33\%, cardiopatia 19,05\%) e 83\% tratavam previamente. Sobre o conhecimento que as comorbidades podem acarretar o AVE, 52,38\% sabiam sobre o assunto. AVEH com 85,71\% de alta e AVEI 57,14\% de óbito. A média de Internação foi: AVEI 24,64 dias e AVEH 14,14 dias. Conclusão: Esta pesquisa encontrou tendências de acordo com a literatura, evidenciando incidência de AVE do tipo isquêmico, suscetíveis em maiores de 60 anos de ambos os sexos, com uma ou mais DCNT e evidenciaram a importância da RAS no acesso à atenção em saúde.
\end{abstract}

Palavras-chave: Acidente Vascular Encefálico; Isquemia cerebral; Hemorragia subaracnóidea; Cuidados críticos; Redes de atenção em saúde.

\begin{abstract}
General purpose: To describe the incidence and profile of the population affected by stroke admitted to the adult intensive care unit (ICU) of a Public Hospital in the city of São Paulo. Methods: Cross-sectional, quantitative, analytical and prospective research. Data collection performed through a questionnaire, information from medical records and completed by the patient or guardian. The sample selected for convenience, composed of 21 individuals hospitalized in the ICU with a diagnosis of stroke, from august to october 2021. Results: 261 patients were hospitalized, $21(8.17 \%)$ with a diagnosis of stroke; 14 (66.67\%) with EVA, male (57.14\%), 7 (33\%) with EVA, female $(57.14 \%)$, both were prevalent in people over 60 years old (76\%), of the white race (61\%). 61\% of patients with previous health follow-up, prevalent in the public health service $(85 \%)$. The highest demand for health service prior to hospitalization was the IS (64.28\%). Among the strokes, 18 had comorbidities (SAH $66.67 \%$, smoking $42.46 \%$, DM $33.33 \%$, heart disease $19.05 \%$ ) and $83 \%$ were previously treated. Regarding the knowledge that comorbidities can lead to CVA, $52.38 \%$ knew about the subject. EVA with $85.71 \%$ of discharge and EVA $57.14 \%$ of death. The average hospitalization days were: AVEI 24.64 days and AVEH 14.14 days. Conclusion: This research found trends in accordance with the literature, showing the incidence of ischemic stroke, susceptible in people over 60 years of both sexes, with one or more CNCDs, and it has evidenced the importance of RAS to the health care system. Keywords: Stroke; Cerebral Ischemia; Subarachnoid hemorrhage; Critical care; Health care networks.
\end{abstract}




\begin{abstract}
Resumen
Objetivo general: Describir la incidencia y el perfil de la población afectada por accidente cerebrovascular internada en la unidad de cuidados intensivos (UCI) de adultos de un Hospital Público de la ciudad de São Paulo. Métodos: Investigación transversal, cuantitativa, analítica y prospectiva. Recogida de datos realizada mediante un cuestionario, información de la historia clínica y cumplimentado por el paciente o tutor. La muestra seleccionada por conveniencia, compuesta por 21 individuos hospitalizados en UCI con diagnóstico de ictus, entre agosto y octubre de 2021. Resultados: Se hospitalizaron 261 pacientes, 21 (8,17\%) con diagnóstico de ictus; $14(66,67 \%)$ con EVA, masculino (57,14\%), 7 (33\%) con EVA, femenino (57,14\%), ambos fueron prevalentes en mayores de 60 años (76\%), de raza blanca (61\%). 61\% de pacientes con seguimiento de salud previo, frecuente en el servicio público de salud (85\%). La mayor demanda del servicio de salud previo a la hospitalización fue el SI (64,28\%). Entre los ictus, 18 presentaban comorbilidades (HAS 66,67\%, tabaquismo 42,46\%, DM 33,33\%, cardiopatía 19,05\%) y el 83\% estaban tratados previamente. En cuanto al conocimiento de que las comorbilidades pueden conducir al ictus, el 52,38\% conocía el tema. EVA con $85,71 \%$ de alta y EVA 57,14\% de muerte. La estancia media fue: AVEI 24,64 días y AVEH 14,14 días. Conclusión: Esta investigación encontró tendencias de acuerdo con la literatura, mostrando la incidencia de accidente cerebrovascular isquémico, susceptible en personas mayores de 60 años de ambos sexos, con una o más ECNT, y evidenció la importancia de la RAS en el acceso a la atención de salud.

Palabras clave: Accidente cerebrovascular; Isquemia cerebral; Hemorragia subaracnoidea; Cuidados críticos; Redes de atención de salud.
\end{abstract}

\title{
1. Introdução
}

O Acidente Vascular Encefálico (AVE) é caracterizado como uma lesão neurológica aguda, de origem vascular com início súbito e rápido. Causada por uma interrupção do fluxo sanguíneo para áreas do encéfalo, de origem isquêmica ou hemorrágica (Medeiros et al., 2017). De acordo com Vieira et al,. (2017), o AVE pode levar à disfunções sensoriais e motoras, sendo uma das maiores causas de mortalidade.

O acidente vascular encefálico isquêmico (AVEI) é decorrente de uma oclusão vascular que gera uma insuficiência do fornecimento sanguíneo, causando uma interrupção de oxigênio e glicose no tecido cerebral, que pode ser por uma obstrução proximal de uma artéria por um trombo, êmbolo ou compressão por um tumor, afetando os processos metabólicos (Gouveia et al., 2015; Medeiros et al., 2017). Até a década 70, a classificação de AVEI era somente baseada em estudos clínicos e na realização de autópsia. Com a evolução da tecnologia, os exames de tomografia cerebral computadorizada, angiografia e mais recente a ecocardiografia passaram a fazer parte da conclusão do diagnóstico (Radu et al.,2017).

Já o acidente vascular encefálico hemorrágico (AVEH) é o rompimento de um vaso sanguíneo e tem como o aneurisma sacular uma das causas mais comuns de lesão, depois as malformações arteriovenosas (MAV's) ou de traumas dentro das áreas extravasculares do cérebro. Com o rompimento do vaso, pode se ter a hemorragia subaracnóidea (HSA), representando de 5 a 10\% de todo o AVE ou hemorragia intraparenquimatosa (Gouveia et al., 2015; Medeiros et al., 2017).

Dentro dos fatores de risco para a ocorrência do AVE, estão os não modificáveis como gênero, raça, história familiar e idade. Já os modificáveis, os quais com a realização de ajuste ao estilo de vida incluindo o acompanhamento e o tratamento em saúde, podem ser reduzidos ou eliminados, entre eles estão as doenças crônicas não transmissíveis (DCNT), como a hipertensão arterial sistêmica (HAS), diabetes melitus (DM), dislipidemia, obesidade, tabagismo, etilismo, sedentarismos, uso de anticoncepcionais orais, doença cardiovascular prévia e cardiopatia isquêmica, stress, condições de saúde mental (Rodrigues et al., 2017).

O AVE pode deixar sequelas que variam de gravidade e característica, como seu tipo, localidade cerebral e extensão. Quando se fala sobre o tipo de AVE, os indivíduos acometidos pelo tipo isquêmico geralmente têm uma maior taxa de sobrevivência em relação ao hemorrágico (Kalaria et al., 2016).

Segundo Sartoretto et al., (2019), quando uma pessoa tem uma suspeita de AVE, aliando os sintomas e os fatores de risco da doença que a predispõe a desenvolvê-la, é preciso procurar um serviço de saúde o mais rápido possível e como recomendação, realizar o exame de tomografia computadorizada de crânio quando possível, que pode detectar se está tendo o evento e classificá-lo em hemorrágico ou isquêmico. Caso a hipótese de AVEH seja descartada e seja confirmada a hipótese 
de AVEI, umas das principais condutas tomadas pelo médico responsável é a terapia trombolítica, a qual tem como objetivo desfazer o trombo e voltar a fornecer o fluxo sanguíneo cerebral tentando minimizar as sequelas causadas por essa oclusão.

O tempo do início dos sintomas é muito importante para a terapia trombolítica, cuja indicação segundo o estudo de Sartoretto et al., (2019), apresenta como tempo limite para alcançar o efeito benéfico de até 4,5 horas do início dos sintomas. Conhecido esse intervalo de tempo como delta $\mathrm{T}$ como um dos principais fatores para a elegibilidade da terapia medicamentosa com trombolíticos. Para a sua aplicação, existem as contraindicações absolutas como, por exemplo, a hipertensão descontrolada e as contraindicações relativas, como idade acima de 75 anos, AVEI severo como coma, entre outros.

Em sua fase aguda, pela instabilidade do quadro, os pacientes muitas vezes necessitam de internação hospitalar em uma unidade de terapia intensiva (UTI), com monitorização contínua. A maioria dos indivíduos que vão para esse setor estão em estado grave e pela função respiratória ineficaz, perda súbita da consciência ou com rebaixamento no nível de consciência, assim necessitando de intubação e de ventilação mecânica invasiva (VMI) para a proteção neurológica e proteção das vias aéreas (Pissolato \& Fleck, 2018).

Segundo Petrolini et al., (2017), é recomendado o tratamento oportuno por meio da reabilitação física na fase intrahospitalar realizado por uma equipe multiprofissional com atuação interdisciplinar de assistente social, enfermeiro, fisioterapeuta, fonoaudiólogo, médico, nutricionista, psicólogo, terapeuta ocupacional, que visa à mobilização, a prevenção de contratura com exercícios passivos e mudanças posturais, deglutição e alimentação, treino de cognição, equilíbrio e das Atividades de Vida Diária (AVD), para conseguir lidar com essa nova realidade, se adaptar com as sequelas que o AVE pode ocasionar, é necessário um apoio psicológico para os indivíduos e seus familiares. Com essas intervenções de maneira oportuna, é possível ajudar a evitar o desenvolvimento de maiores complicações.

No Brasil, segundo as Diretrizes de Atenção à Reabilitação da Pessoa com Acidente Cerebral (2013), o AVE tem como incidência 108 casos para mil habitantes com taxa de fatalidade aos trinta dias de $18.5 \%$ e aos 12 meses de 30,9\%3․ Medeiros et al., 2017, aponta que no Brasil o AVE ainda é uma das principais causas de morte e que em projeções para o ano de 2015 a 2030 a incidência de AVE expressa números alarmantes, com expectativa de 18 e 23 milhões de novos casos no mundo.

De acordo com a World Stroke Organization (Organização Mundial de AVE) um a cada seis indivíduos no mundo terá AVE ao longo da sua vida. Com essas informações, o Ministério da Saúde enfatiza a importância de ações voltadas à vigilância à saúde visando não só a reabilitação dos indivíduos acometidos, como também a prevenção e promoção de saúde para melhorar a qualidade de vida de toda população (Ministério da Saúde, 2013).

Segundo a Secretaria de Vigilância em Saúde, no Brasil, em um período de 10 meses em 2020, aproximadamente 79 mil pessoas morreram em decorrência de AVE, demonstrando alta taxa de letalidade (SP, 2020). Já no município de São Paulo, em 2014 o AVE foi responsável pela morte de 5.665 pessoas e outras 10.704 pessoas foram internadas com esse diagnóstico (SP, 2015).

Conforme dados da assistência hospitalar mais recente do Boletim de Saúde da Coordenação de Epidemiologia e Informação (CEInfo2021), dados do município de SP, as internações ocorridas em estabelecimentos SUS sob gestão municipal com o diagnóstico principal de doenças do aparelho circulatório em 2019 chegou a 26.633 internações no ano correspondendo a 34,9\% dos outros diagnósticos, levando em média de permanência de internação 7.3 dias.

Kaup et al., (2015), realizou uma pesquisa no período de 2004 a 2010 sobre a taxa de mortalidade de indivíduos com diagnóstico de AVE na região de São Paulo e concluiu que a mortalidade é maior em regiões com um menor índice de Desenvolvimento Humano (IDH). Em São Paulo, na região da zona Leste, a taxa de mortalidade por AVE por 100.000 habitantes foi de $77,8 \%$. O autor propõe como justificativas possíveis, a falta de acesso às informações, a estrutura (física e humana) de atendimento entre outras. 
A prevalência do AVE ainda é alta, segundo o Governo Federal do Estado de São Paulo, em 2017, as doenças cerebrovasculares foram a segunda causa de morte no Brasil. Entre os sobreviventes, $90 \%$ apresentam alguma deficiência ou incapacidade, caracterizada como prejuízos nas funções sensitivas, motoras, do equilíbrio, da marcha, de déficit cognitivos, de alterações de fala e linguagem e disfagia; $15 \%$ dos pacientes não apresentam prejuízo da capacidade funcional, porém $85 \%$ necessitam de reabilitação física, cognitiva, psicossocial e cuidado constante, o que pode levar a um impacto econômico, social e familiar significativo. As repercussões e sequelas deixadas pelo AVE geram restrições e limitações nas (AVD) e na participação social (Medeiros et al., 2017; Vieira et al.,2017).

As Redes de atenção à Saúde (RAS) têm o objetivo de promover a integração sistêmica dos cuidados em saúde, propondo a organização dos serviços em níveis de complexidade diferente, de forma a contribuir para a continuidade da atenção à saúde para a população A melhor compreensão das condições crônicas e agudas da população atreladas à promoção do cuidado multidisciplinar, a melhora do acesso à atenção contínua e integral de qualidade, responsável e humanizada, respeitando os princípios da equidade, eficácia clínica, sanitária e eficiência econômica, podem levar a melhora na assistência sistêmica do cuidado em saúde enfatizando a importância da RAS (Arruda et al., 2015).

A sistematização da RAS se organiza em eixos temáticos ou de acordo com as condições de saúde da população com o ciclo da vida. A RAS se dá pela composição de três elementos que a constitui, a saber: população, estrutura operacional e modelos de atenção à saúde. A estrutura operacional é constituída pela interligação das Redes que levam à qualificação, educação, informação, regulação, promoção e vigilância à saúde, entre elas, sendo o elemento que mais se encaixa quando se há pacientes internados em uma UTI e precisam pensar no plano de alta, com a Rede de Atenção às Urgências e Emergências, Rede de Atenção Psicossocial, Rede de Atenção às doenças e condições crônicas, Rede de Cuidado à Pessoa com Deficiência e por fim a Rede Cegonha (Arruda et al., 2015; Almeida et al., 2015; Diretrizes, 2014).

Os modelos de atenção à saúde organizam-se em graus crescentes de complexidades na atenção à saúde e promoção do cuidado integral por meios de fluxos entre os serviços de saúde coerentes e articulados entre si com os pontos estratégicos da Atenção Primária à Saúde, à Atenção Ambulatorial Especializada, a Atenção Hospitalar Especializada, a Urgência e a Emergência, com destacada importância, a articulação Intersetorial (Arruda et al., 2015; Almeida et al., 2015; Diretrizes, 2014). De acordo com Mendes (2011), em diversos outros países, a organização de serviços em rede demonstrou melhores resultados, como maior satisfação de usuários, redução no número de internações, melhor uso dos recursos entre outras vantagens.

Com o grande número de internações de pacientes com o diagnóstico de AVE sendo isquêmico ou hemorrágico, descrito nas evidências científicas e no setor de Terapia Intensiva Adulta do Hospital Municipal Dr. Carmino Caricchio, despertou-se o interesse para desenvolvimento dessa pesquisa. Conhecido como Hospital Tatuapé, localiza-se na cidade de São Paulo, capital do estado que possui 44.884 .143 habitantes (junho de 2021), sendo o estado mais populoso do Brasil. A cidade de São Paulo com 12.325.232 pessoas e especificamente no bairro do Tatuapé, que possui aproximadamente 91 mil habitantes. Com mais de 50 anos, o Hospital é referência de atendimento de alta complexidade para Coordenadoria de Saúde Sudeste; uma região populosa com aproximadamente 2.666 .027 habitantes em 2015. A coordenadoria Sudeste é composta por cinco Supervisões Técnicas de Saúde, entre elas a Vila Mariana, Ipiranga, Vila Prudente/Sapopemba, Mooca/Aricanduva/Carrão e Penha. (SP, 2019; SEADE, 2021, COSEMSSP, 2015).

A Coordenadoria de Saúde Sudeste, em março de 2021, contou com 7 hospitais municipais de serviços próprios da Secretaria Municipal da Saúde e com os dados do CEInfo, em 2019, essa mesma região teve como coeficiente de mortalidade das doenças cerebro-vasculares um valor de 34,6\% e umas das DCNT abordadas, como a Diabete Mellitus com o valor de 15,2\% (SP, 2020; CEINFO, 2021). 
O Hospital Municipal do Tatuapé é um hospital público geral de grande porte, nível terciário, com mais de oito especialidades, pertencendo a Rede de Atenção às Urgências e Emergências, Rede de Atenção às doenças e condições crônicas, que atende aproximadamente trinta mil pacientes por mês, com UTI adulto, pediátrica e neonatal, a unidade possui uma Unidade de Pronto Atendimento (UPA) sob gestão da Associação Paulista para o Desenvolvimento de Medicina (SPDM) (SP, 2019).

A unidade hospitalar conta com o manejo inicial do AVE, sendo o primeiro atendimento realizado e após o quadro clínico estabilizar se for possível para a alta hospitalar. Para que isso ocorra deve seguir os planos de alta para casos de AVEI e AVEH, com as informações completas contendo o diagnóstico e informação médica relevante, receita médica completa, indicação dos tratamentos especializados de equipe multidisciplinar de que o paciente necessitará, bem como a avaliação para a indicação de atenção domiciliar, (Serviço de Atenção Domiciliar -SAD), indicação de necessidades especiais como cadeira de roda e órteses, encaminhamento à unidade de Atenção Primária de referência para coordenação do cuidado e prevenção secundária, objetivos a serem compartilhados com a reabilitação seguindo as RAS (Brasil, 2020).

Nessa circunstância, o presente estudo tem com o objetivo principal de descrever a incidência e o perfil da população internada com diagnóstico de primeiro evento de AVE na UTI. Bem como verificar o nível de consciência após o evento, a necessidade de suporte ventilatório e dias totais de internação, verificar a aplicação da terapia trombolítica no primeiro atendimento hospitalar e o conhecimento dos pacientes internados e se necessário dos seus responsáveis sobre as doenças crônicas não transmissíveis (DCNT) que podem causar o AVE e a adesão aos processos de cuidado em saúde ofertados na atenção primária, relacionando com acompanhamento/tratamento das DCNT.

\section{Metodologia}

Estudo transversal, quantitativo, analítico, prospectivo realizado no período de agosto a outubro de 2021 em um hospital público da Coordenadoria de Saúde Sudeste, pertencente à Secretaria Municipal da Saúde de São Paulo. Foram incluídos indivíduos com diagnóstico de primeiro evento de AVE isquêmico ou hemorrágico que aceitaram participar da pesquisa ou que o responsável autorizou, com idade superior a 18 anos, independente do gênero, em ventilação mecânica ou não, internados na Unidade de Terapia Intensiva Adulta do Hospital Municipal Dr Cármino Caricchio. Foram excluídos indivíduos que apresentaram AVE prévio ou acometido no período de internação, prontuários incompletos e aqueles que não assinaram o Termo de Consentimento Livre e Esclarecido. Houve aprovação do Comitê de Ética (n ${ }^{\circ}$ 5.207.079) e assinatura do Termo de Consentimento Livre e Esclarecido (TCLE), os dados foram coletados dos prontuários dos pacientes e quando necessário completado pelos pacientes ou responsáveis em um questionário estruturado que constavam os dados gerais de identificação, perguntas especificas sobre o estado geral de saúde e por fim sua evolução pós UTI e o tempo de internação na mesma.

A coleta de dados foi realizada pela fisioterapeuta/ pesquisadora durante o período de internação. Ao entrar em contato com o paciente/responsável, a fisioterapeuta se identificou e explicou brevemente os objetivos da pesquisa e entregou ou leu o TCLE. Após concordância dos pacientes/responsáveis em participar da pesquisa e assinatura do TCLE, iniciou-se a coleta. Os dados foram analisados e aplicadas médias, medianas, desvio padrão, testes paramétricos e/ou testes não paramétricos levandose em consideração a natureza da variável estudada. Para os fatores de risco foi aplicado, o Teste G de Cochran e também o teste do Qui-quadrado para confronto dos grupos estudados, os dados coletados foram armazenados no software aplicativo Microsorft ${ }^{\circledR}$ Excel $®$ em uma planilha.

\section{Resultado}

Durante o período de agosto a outubro de 2021 (60 dias) foram internados 261 pacientes com diversos diagnósticos na UTI adulto, desses, 25 (9,57\%) com o diagnóstico de AVE, 04 foram excluídos (03 possuíam AVE prévio e 01 recusou participar), 
a amostra final foi composta por $21(8,17 \%)$ pacientes, sendo $14(66,67 \%)$ com AVEI e $7(33,33 \%)$ com AVEH. A composição da amostra está demonstrada na Figura 1.

Figura 1: Composição da amostra.

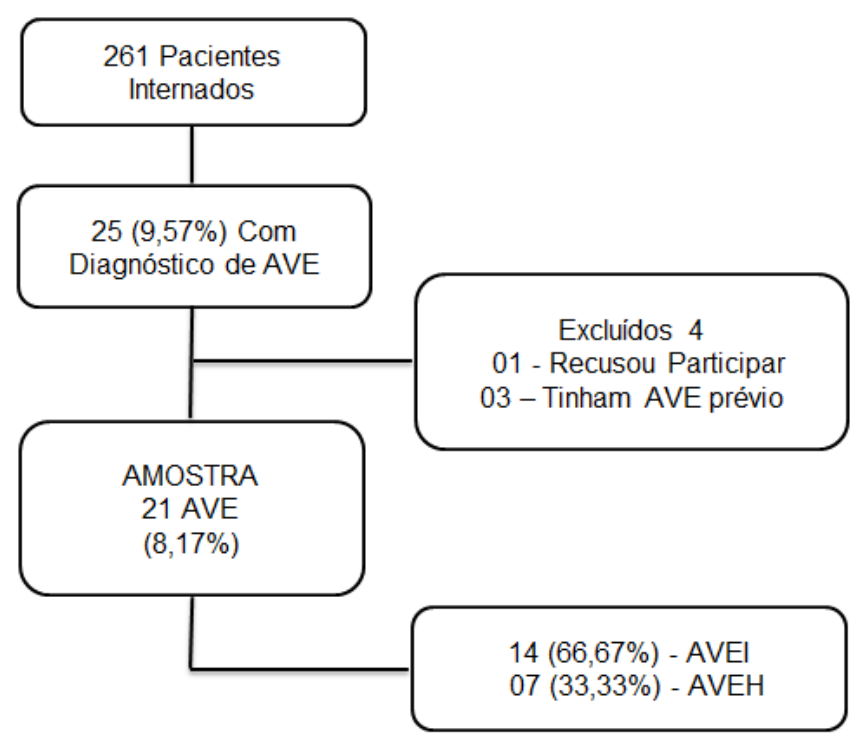

Fonte: Autores (2021).

A prevalência de internação por AVE foi de 9,57\% maior parte da amostra foi composta por pacientes com diagnóstico de AVEI, totalizando $14(66,67 \%)$ sendo 57,14\% do sexo masculino, em seguida por pacientes com diagnóstico de AVEH $(33,33 \%)$ com maior prevalência no sexo feminino (57,14\%). A idade média de todos os acometidos foi de 64,3 anos, com maior incidência de acometimento na raça branca totalizando $13(61,90 \%)$ pacientes, conforme mostra o Quadro 1.

Quadro 1: Perfil dos pacientes acometidos por AVE.

\begin{tabular}{|c|c|c|c|}
\hline \multirow{2}{*}{ Item } & \multirow{2}{*}{ Critério } & A VEI & A VEH \\
\hline & & $\mathbf{N}(\%)$ & $\mathbf{N}(\%)$ \\
\hline \multirow[t]{2}{*}{ Gênero } & Masculino & $8(57,14 \%)$ & $3(42,85 \%)$ \\
\hline & Feminino & $6(42,85 \%)$ & $4(57,14 \%)$ \\
\hline Idade & Média & \multicolumn{2}{|l|}{64,3} \\
\hline \multirow[t]{3}{*}{ Raça } & Branca & $9(64,28 \%)$ & $4(57,14 \%)$ \\
\hline & Parda & $2(14,28 \%)$ & $1(14,28 \%)$ \\
\hline & Negro & $3(21,42 \%)$ & $2(28,57 \%)$ \\
\hline
\end{tabular}

Fonte: Autores (2021).

Quando abordado sobre o acompanhamento prévio da saúde, a maior parte dos acometidos por AVEI (71,42\%) referiram realizar acompanhamento de saúde. O resultado foi oposto nos casos onde o acometimento foi por AVEH. Dos 07 
acometidos, maior parte relatou não fazer acompanhamento, desses, 03(42,25\%) faziam acompanhamento prévios sendo estes do sexo feminino, os outros 04 que não realizavam acompanhamento, 03 eram homens e 01 mulher. Sobre o serviço de saúde procurado, $18(85,21 \%)$ participantes afirmaram que utilizam serviços públicos de saúde, conforme o quadro 2.

Quando abordados sobre as complicações das DCNT, e que estas poderiam causar AVE, $6(42,85 \%)$ pacientes com AVEI e $5(71,42 \%)$ com AVEH relataram ter ciência dessas complicações, totalizando 52,38\%. 12 (57,14\%) pacientes informaram ter procurado serviços de saúde no mês anterior à internação, destes, 09 (75,00\%) tiveram AVEI e 03 (25,00\%) AVEH. 08(66,67\%) pacientes procuraram a atenção primária (UBS) e $04(33,33 \%)$ o pronto socorro (PS), desses, 02 (50,00\%) sofreram complicações Isquêmicas e 02 (50,00\%) Hemorrágicas. 02 pacientes referiram internação prévia, nos últimos 12 meses, conforme o Quadro 2.

Quadro 2: Acompanhamento, serviço acessado e ciência sobre as DCNT.

\begin{tabular}{|c|c|c|c|}
\hline \multirow{2}{*}{ Item } & \multirow{2}{*}{ Critério } & A VEI & A VEH \\
\hline & & $\mathbf{N}(\%)$ & $\mathbf{N}(\%)$ \\
\hline \multirow[t]{3}{*}{$\begin{array}{l}\text { Acompanhamento } \\
\text { prévio de saúde }\end{array}$} & Realizavam & $10(71,42 \%)$ & $3(42,25 \%)$ \\
\hline & Serviço Público & $13(92,85 \%)$ & $5(71,42 \%)$ \\
\hline & Serviço Privado & $1(71,14 \%)$ & $2(28,57 \%)$ \\
\hline Ciência & & $6(42,85 \%)$ & $5(71,42 \%)$ \\
\hline \multirow[t]{3}{*}{$\begin{array}{c}\text { Procuraram serviço } \\
\text { de saúde }\end{array}$} & & $9(64,28 \%)$ & $3(42,85 \%)$ \\
\hline & $\begin{array}{l}\text { Atenção } \\
\text { básica }\end{array}$ & $7(77,77 \%)$ & $1(33,33 \%)$ \\
\hline & Pronto socorro & $2(22,22 \%)$ & $2(66,66 \%)$ \\
\hline Internação prévia & & $2(14,28 \%)$ & 0 \\
\hline
\end{tabular}

Fonte: Autores (2021).

Grande parte dos pacientes referiram realizar acompanhamento para DCNT, as doenças mais prevalentes por participantes foram hipertensão arterial sistêmica (HAS) 66,67\%, tabagismo 42,86\%, diabete mellitus (DM) 33,33\%, cardiopatia $19,05 \%$ e outros $47,62 \%$ como obesidade, câncer, etilismo. Desta forma $18(85,71 \%)$ tinham uma ou mais DCNT, conforme mencionado no Gráfico 1. 
Gráfico 1: Prevalências das DCNT nos pacientes internados com AVE.

$100,00 \%$

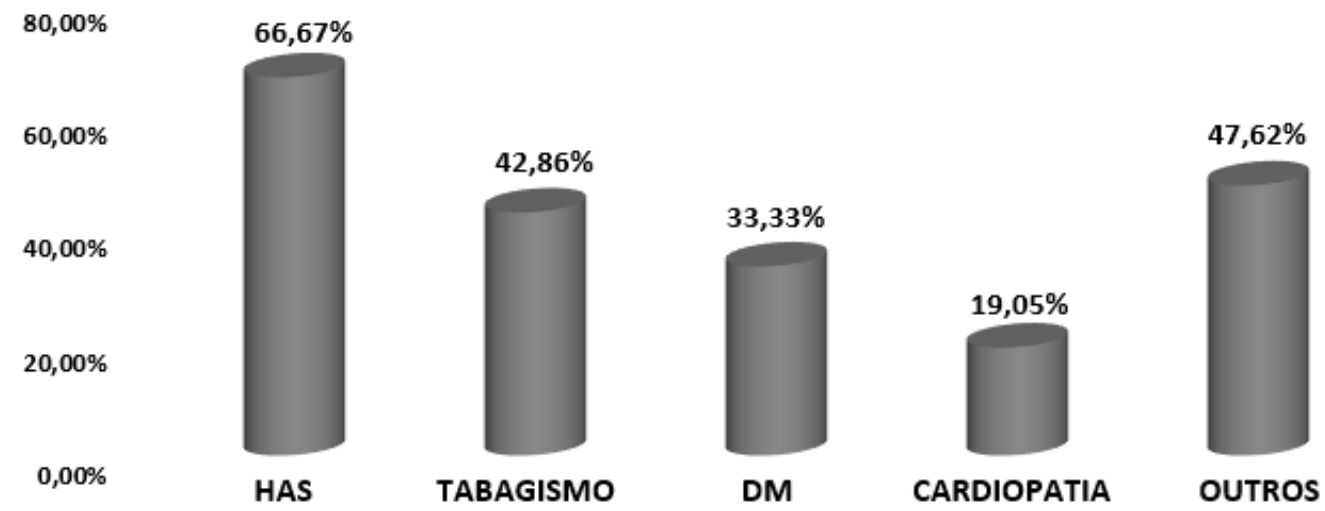

Fonte: Autores (2021).

No primeiro dia de internação, encontravam-se 10 (47,61\%) pacientes sedados, 06 (60,00\%) com AVEI e 04 (40\%) com AVEH. Logo após o evento inicial de AVE, 13 (61,90\%) pacientes com intubação orotraqueal na UTI, sendo 09 (69,23\%) com AVEI e 04 (30,77\%) com AVEH. Dos 14 pacientes com AVEI, 03 (21,43\%) foram trombolizados, destes 02 (66,67\%) evoluíram a óbitos e $1(33,33)$ recebeu alta em 18 dias, conforme a tabela 3.

Dos 14 pacientes internados na UTI com AVEI, 08(57,14\%) foram a óbito, (71,43\% equivalente a 05 mulheres e 37,50\% equivalente a 03 homens) e $06(42,86 \%)$ tiveram alta da unidade, dos 07 com AVEH, 06(85,71\%) tiveram alta da unidade e $01(14,29 \%)$ foi a óbito (mulher). A média de dias de internação dos pacientes no geral foram 21,14 $( \pm 0,41)$ dias, com AVEI foram 24,64 ( $\pm 0,45)$ dias e com AVEH foram 14,14 $( \pm 0,75)$ dias, conforme o Quadro 3.

Quadro 3: Desfecho do processo de internação.

\begin{tabular}{|c|c|c|c|}
\hline \multirow{2}{*}{ Item } & \multirow{2}{*}{ Critério } & A VEI & AVEH \\
\hline & & $\mathbf{N}(\%)$ & $\mathrm{N}(\%)$ \\
\hline Sedação & & $6(42,85 \%)$ & $4(57,14 \%)$ \\
\hline Intubação orotraqueal & & $9(64,28 \%)$ & $4(57,14 \%)$ \\
\hline \multicolumn{4}{|l|}{ Evolução } \\
\hline Alta & & $6(42,86 \%)$ & $6(85,71 \%)$ \\
\hline \multirow[t]{3}{*}{ Óbito } & & $8(57,14 \%)$ & $1(14,29 \%)$ \\
\hline & Homens & $3(37,50 \%)$ & 0 \\
\hline & Mulheres & $5(71,43 \%)$ & $1(14,29 \%)$ \\
\hline Trombolizado & & $3(21,42 \%)$ & NA* \\
\hline $\begin{array}{c}\text { Média de Dias de } \\
\text { Internação }\end{array}$ & & $24,64( \pm 0,45)$ & $14,14( \pm 1,08)$ \\
\hline
\end{tabular}

*NA: não se aplica. Fonte: Autores (2021). 
Os dados desta pesquisa foram submetidos à análise estatística sendo utilizado o teste de $\mathrm{G}$ de Cocharan e o teste Quiquadrado, sem resultados significativos.

\section{Discussão}

Este estudo identificou a internação dos pacientes com o primeiro episódio de AVE, tendo a sua maior incidência do AVE isquêmico (66,67\%) seguido do hemorrágico (33,33\%) em indivíduos com a média de idade de 64,3 anos. Esses resultados estão de acordo com a literatura, segundo Schimidt et al., (2019), 85\% dos casos, o AVE ocorre por isquemia de forma transitória ou permanente e 15\% dos casos são AVE hemorrágico.

Quando relacionado a idade média encontrada na pesquisa, também corrobora com achados de outros estudos, como o da Oliveira \& Waters (2021), que acaba concluindo que o AVE tem sua principal incidência em uma população idosa com idade superior há 60 anos, justificado por essa população na maioria das vezes possuírem mais comorbidades associadas, os quais são fatores de risco para o AVE.

Em relação ao sexo mais acometido no AVEI, 57,14\% foram no sexo masculino e AVEH, mesmo com o menor número de casos, a prevalência foi em 57,14\% no sexo feminino, sendo no geral dos dois $11(52,38 \%)$ pacientes homens e $10(47,61 \%)$ pacientes mulheres. Esses dados corroboram com o resultado no estudo de Schimidt et al., (2019), em que a proporção de casos entre os sexos masculino e feminino foram quase iguais em sua pesquisa. Já em relação à raça mais acometida temos maior incidência na raça branca 13(61,90\%), seguido da raça negra, 05 (23,80\%) e pardo 03 (14,28\%), sendo compatível com o estudo de Roxa et al., 2021, que evidencia que a raça branca pode ser um fator de risco provável para o AVE.

Sobre o acompanhamento prévio da saúde, antes da internação por AVE. A maior parte dos acometidos pelo AVEI (71,43\%) relataram que "sim" realizavam acompanhamento. Já os acometidos por AVEH, 3 (42,86\%) referiram acompanhar, 4 negaram acompanhamento, sendo 03 do sexo masculino e 01 do sexo feminino, demonstrando uma menor adesão ao tratamento de indivíduos do sexo masculino. Esses dados mostram que o preconizado por Brasil et al., (2014), no Caderno de Atenção Básica com a Estratégias para o Cuidado da Pessoa com Doença Crônica vem surgindo efeitos positivos que promovem autonomia e autocuidado, estimulados pelo profissional de saúde para que o individuo se torne o sujeito da ação na promoção de saúde.

Em relação a qual serviço de saúde procuram para fazer acompanhamento de saúde, a maioria dos pacientes $(85,21 \%)$, responderam que utilizam os serviços públicos de saúde. Vale ressaltar que $12(57,14 \%)$ relataram ter procurado os serviços de saúde no último mês antes da internação, destes, 09 no período da pesquisa tiveram seu primeiro episódio de AVEI e 03 AVEH. Dentre esses 12 que procuraram o serviço de saúde, 08 pacientes procuraram a atenção básica, 04 o pronto socorro (02 sofreram complicações Isquêmicas e 02 Hemorrágicas) e 02 pacientes referiram internação prévia, nos últimos 12 meses. Podendo esta procura servir como um sinal de alerta, para o possível agravamento dos sintomas das DCNT, esta pesquisa teve como objetivo identificar o acompanhamento prévio em saúde e se o indivíduo procurou os serviços de saúde antes da internação.

Os resultados desta pesquisa evidenciaram a importância da RAS na promoção do acesso à atenção em saúde, demonstrando que a grande maioria da população internada nesta instituição referiu que foram atendidas no sistema público de saúde e afirmaram que utilizavam o Sistema Único de Saúde para realizar o acompanhamento e o monitoramento das DCNT. Vale enfatizar que estudo de Mendes (2011) e Arruda et al., (2015), explicam o funcionamento das RAS que tem como objetivo a promoção, proteção e recuperação da saúde e possui como um dos principais pontos de atenção a tentativa de reduzir o número de internações. Após esse período, vale reforçar a importância não somente da Rede de Atenção às Urgências e Emergências e sim também da Rede de Atenção às doenças e condições crônicas, Rede de Cuidado à Pessoa com Deficiência e a Rede de Atenção Psicossocial para que assim esse paciente possa seguir seu tratamento de forma integral. 
Quando observados os fatores de risco modificáveis do AVE, nessa pesquisa foram encontrados as DCNT com a de maior frequência a HAS $(66,67 \%)$, seguido do tabagismo (42,86\%), DM (33,33\%), cardiopatia $(19,05 \%)$, e outros $(47,62 \%)$ como obesidade, câncer, etilismo. Segundo o estudo de Roxa et al. (2021), as DCNT mais prevalentes no AVE são HAS (68,9\%), dislipidemia (29,00\%), DM (26,70\%), cardiopatias (22,70\%) e o tabagismo que evidenciou alto risco. Identificou-se que pacientes mais velhos têm maiores incidência e piores evoluções de AVE e com a maior taxa de mortalidade na fase aguda do AVE e a longo prazo, pela influência também do perfil cardiovascular, socioeconômico, além do tipo de AVE. Lembrando que no estudo atual no qual já foi citado que a prevalência do AVE na UTI foi em pacientes com mais de 60 anos, teve a maior incidência de óbitos no do tipo isquêmico chegando a 57,14\%.

A amostra foi composta por 21 pacientes, 03 (14,28\%) informaram que não realizavam acompanhamento por DCNT, os quais foram pegos de surpresa pelo diagnóstico. Os outros 18 (85,71\%), referiram ter alguma DCNT e faziam o acompanhamento/tratamento da mesma, entre esses, 03 (16,66\%) pacientes não faziam nenhum tipo de tratamento. Foram abordados todos os pacientes ou até mesmo seus responsáveis sobre as complicações das DCNT, e que estas poderiam causar um AVE e um pouco mais que a metade, 11 pacientes $(52,38 \%)$ relataram ter ciência dessas complicações tendo de alguma maneira acesso as informações de saúde.

Esses resultados encontrados na pesquisa não estão de acordo com o resultado do estudo realizado por Kaup et al., (2015), refere que uma das justificativas que tornam o AVE mais letal em regiões mais humildes como na zona leste São Paulo (SP) é a falta ou dificuldade do acesso à informação e a estrutura (física e humana) de atendimento. Sendo que a pesquisa atual foi realizada em um hospital localizado na zona leste de SP e sua coordenadoria de saúde informa que até o dia de hoje existe 7 hospitais municipais de serviços próprios da secretaria municipal de saúde. Mas vale ressaltar que já se passaram seis anos do estudo de KAUP et al 2015 até a presente pesquisa e com isso melhorias puderam ter sido sinalizadas colaborando com a ampliação do acesso e cuidado em saúde.

Durante a coleta de dados para essa pesquisa, no primeiro dia da avaliação foram encontrados 10 (47,61\%) pacientes sedados (60,00\% com AVEI e 40,00\% com AVEH) e 13(61,90\%) pacientes com intubação orotraqueal, (69,23\%) com AVEI e 30,77 com AVEH). De acordo com Frazão et al., (2020), pacientes com o diagnóstico inicial de AVE necessitam de uma monitorização contínua dos sinais vitais e do nível de consciência. Quando apresentam alterações como o rebaixamento do nível de consciência levando a uma pontuação menor que 8 na escala de coma de Glasgow ou insuficiência respiratória e até mesmo risco de broncoaspiração, deve ser avaliada a realização de sedação e de intubação orotraqueal (IOT) para a proteção neurológica e proteção das vias áreas.

Não foi pesquisado o motivo pelo qual optou-se pela utilização da sedação e da IOT como estratégia clínica, mas podese notar que a maioria dos casos do uso de sedação e da IOT foi nos pacientes acometidos com AVEI, podendo ser justificado por ter sido em maior prevalência na pesquisa. Segundo o estudo de Frazão el at., (2020), 20\% dos pacientes que deram entrada na sala de emergência foram com diagnóstico de AVE sem especificar o tipo e foram submetidos a IOT por causa do rebaixamento do nível de consciência, com isso podemos ter a noção da principal causa que pode levar um paciente com esse diagnóstico a IOT.

Quando verificado sobre a terapia de trombólise para os que foram acometidos por AVEI, encontrou-se no estudo 14 pacientes internados e destes, 3 (21,43\%) foram trombolizados, com isso 2 (66,67\%) evoluíram a óbito e 1 recebeu alta em 18 dias. Como não foi possível avaliar os motivos pelo quais os outros 11 não receberam a terapia trombolítica e por ser um número baixo de trombolizados, levantando a hipótese se foi por falta de conhecimento partindo dos próprios pacientes em relação aos sintomas de um AVE para que procurasse o serviço de saúde a tempo, ou por ser contra indicado por algum outro motivo ou se foi por questões relacionadas ao próprio serviço. Estudo como o de Roxa et al., (2021), preconiza o tempo limite entre o início dos sintomas e o início da terapia trombolítica em até 4,5 horas para que consiga restaurar o fluxo sanguíneo 
cerebral evitando possíveis sequelas. Fora desse tempo, pode trazer consequências como a transformação da isquemia em hemorragia.

Foi acompanhada a evolução dos participantes da pesquisa até seu último dia na UTI e como resultado chegou-se a um maior número de óbito do que de alta por AVE isquêmico, sendo que dos 14 pacientes, 8 (57,14\%) foram a óbito, destes 5 $(71,43 \%)$ mulheres e $3(37,50 \%)$ homens, e $6(42,86 \%)$ tiveram alta para a enfermaria. Em relação aos acometidos por AVE hemorrágico, dos 7 pacientes, $6(85,71 \%)$ tiveram alta da unidade e 1 (14,29\%) foi a óbito (mulher). Possivelmente, esse dado também se justifique por os que foram acometidos por AVEI serem em maior número. Os resultados encontrados estão de acordo com a pesquisa de Mamed et al., (2017), em 60 cidades a maioria dos óbitos foram por isquemia (56,3\%) e do sexo feminino com $56 \%$.

Por fim, a média de dias de internação dos pacientes na UTI com AVEI foi de 19,38 $( \pm 0,49)$ dias e AVEH $14,14( \pm$ 0,75) dias, no geral do AVE foi de 17,55 $( \pm 0,63)$ dias. No estudo de Mourao et al., (2017), foi avaliado o maior tempo de internação de AVE na enfermaria e no centro de terapia intensiva chegando a 47,3 dias $( \pm 4,2)$ e 27,5 dias $( \pm 2,5)$ justificando por grande parte dos pacientes que apresentaram complicações na internação.

\section{Considerações Finais}

Embora o número da amostra tenha sido baixo, o que restringiu as possibilidades da análise estatística, esta pesquisa foi capaz de encontrar tendências que estão de acordo com a literatura, evidenciando a incidência de AVE com prevalência do tipo isquêmico, tendo os mais suscetíveis ao acometimento por AVE as pessoas com idade superior a 60 anos de ambos os sexos, com uma ou mais DCNT. Os resultados desta pesquisa evidenciaram, ainda, a importância da RAS no acesso à atenção primária.

Pode-se notar que mais da metade da população do estudo apresenta um grau importante de conhecimento sobre as DCNT e por fim, a média de dias de internação dos pacientes na UTI com AVEI foi de 19,38 $( \pm 0,49)$ dias e AVEH $14,14( \pm$ $0,75)$ dias. Sugere-se a continuidade do trabalho de prevenção e promoção em saúde.

Recomenda-se que novas pesquisas sejam realizadas para o aprofundamento destas informações, com um possível aumento do número amostral e do tempo da pesquisa.

\section{Referências}

Arruda, C., Lopes, S. G. R., Koerich, M. H. A. D. L., Winck, D. R., Meirelles, B. H. S., \& Mello, A. L. S. F. D. (2015). Redes de atenção à saúde sob a luz da teoria da complexidade. Escola Anna Nery, 19, 169-173.

Brasil. (2014). Ministério da Saúde, Secretaria de Atenção à Saúde, \& Departamento de Atenção Básica. Estratégias para o cuidado da pessoa com doença crônica. Cadernos de Atenção Básica, (35).

BRASIL. (2020). Ministério da saúde. Linhas de cuidado do acidente vascular cerebral no adulto (AVC).

http://189.28.128.100/dab/docs/portaldab/publicacoes/LC_AVC_no_adulto.pdf.

CEInfo. (2021). Boletim Saúde da Coordenação de Epidemiologia e Informação.

https://www.prefeitura.sp.gov.br/cidade/secretarias/upload/saude/arquivos/publicacoes/Boletim_CEInfo_Dados_2020.pdf.

COSEMSSP. (2015). Apresentação. Coordenadoria Regional de Saúde Sudeste. http://www.cosemssp.org.br/downloads/Apresentacao-Karina-coordenadoriasul.pdf.

de Sousa Rodrigues, M., Fernandes, L., \& Galvão, I. M. (2017). Fatores de risco modificáveis e não modificáveis do AVC isquêmico: uma abordagem descritiva. Revista de medicina, 96(3), 187-192.

de Oliveira, G. G., \& Waters, C. (2021). Perfil epidemiológico dos pacientes com acidente vascular cerebral isquêmico/Epidemiological profile of patients with stroke. Arquivos Médicos dos Hospitais e da Faculdade de Ciências Médicas da Santa Casa de São Paulo, 66(1u), 1-5.

Frazão, D. A. L., de Andrade, O. G. C., Muniz, G. G., Bächtold, G. A. B., \& de OG Santos, J. R. (2020). Prevalência de intubação orotraqueal no serviço de emergência em hospital secundário do Distrito Federal. Brazilian Journal of Development, 6(6), 39137-39148. 
Gouvêa, D., de Paula Gomes, C. S., de Melo, S. C., do Nascimento Abrahão, P., \& Barbieri, G. (2015). ACIDENTE VASCULAR ENCEFÁLICO: UMA REVISÃO DA LITERATURA. Ciência Atual-Revista Científica Multidisciplinar do Centro Universitário São José, 6(2).

Gomes, C. S. P., Melo, S. C., Abrahão, P. N. \& Barbieri, G. (2015). Acidente vascular encefálico: uma revisão de literatura. Rev. Científica Multidisciplinar das Faculdades São José. 6(2): 2-6.

Helder, H. A. J., do Nascimento, A. F. A., Alves, A. W., Barbosa S. J., Costa, L. L., \& Nunes, A. S. M. (2015). ATENÇÃO PRIMÁRIA À SAÚDE: ENFOCANDO AS REDES DE ATENÇÃO À SAÚDE. Journal of Nursing UFPE/Revista de Enfermagem UFPE, 9(11).

Hoffmann, M. C. C. L., \& Lobo, M. C. D. A. (2014). Diretrizes para o cuidado das pessoas idosas no SUS: proposta de modelo de atenção integral.

Kaup, A. O., Dos Santos, B. F, Victor, E. S., Cypriano, A.S., Lottenberg, C.L., Cendoroglo Neto, M., \& Silva, G.S. (2015). Georreferenciando óbitos por AVC em São Paulo: um cinturão de AVC intramunicipal?. International Journal of Stroke , 10, 69-74.

Kalaria, R. N., Akinyemi, R., \& Ihara, M. (2016). Stroke injury, cognitive impairment and vascular dementia. Biochimica et biophysica acta, 1862(5), 915925.

Mamed, S. N., Ramos, A. M. D. O., Araújo, V. E. M. D., Jesus, W. S. D., Ishitani, L. H., \& França, E. B. (2019). Perfil dos óbitos por acidente vascular cerebral não especificado após investigação de códigos garbage em 60 cidades do Brasil, 2017. Revista Brasileira de Epidemiologia, 22.

Medeiros, C. S. P. D., Silva, O. A. P. D., Araújo, J. B., Souza, D. E. D., Cacho, E. W. A., \& Cacho, R. D. O. (2017). Perfil social e funcional dos usuários da Estratégia Saúde da Família com Acidente Vascular Encefálico. Revista brasileira de ciências da saúde, 21(3), 211-220.

MENDES, E. V., (2011). As redes de atenção à saúde. Organização Pan-Americana da Saúde.

Ministério da Saúde (BR). (2013). Diretrizes de atenção à reabilitação da pessoa com acidente vascular cerebral.

Mourao, A. M., Vicente, L. C. C., Chaves, T. S., SantAnna, R. V., Meira, F. D. C., Xavier, R. M. D. B., ... \& Teixeira, A. L. (2017). Perfil dos pacientes com diagnóstico de AVC atendidos em um hospital de Minas Gerais credenciado na linha de cuidados. Rev Bras Neurol, 53(4), 12-16.

Mateus, A. P., Ruivo, E. A. B., \& Brito, W. A. (2017). Mobilização precoce intra-hospitalar em pacientes após acidente vascular cerebral: revisão sistemática. Arq. Ciênc. Saúde, 08-13.

Radu, R. A., Terecoasă, E. O., Băjenaru, O.A. \& Tiu, C. (2017). Classificação etiológica do AVC isquêmico: Onde estamos? De neurologia clínica e neurocirurgia, 159, 93-106.

Roxa, G. N., Amorim, A. R. V., Caldas, G. R. F., Ferreira, A. D. S. H., de Alencar Rodrigues, F. E., Gonçalves, M. O. S. S., \& da Silva, C. R. L. (2021). Perfil epidemiológico dos pacientes acometidos com AVC isquêmico submetidos a terapia trombolítica: uma revisão integrativa. Brazilian Journal of Development, 7(1), 7341-7351.

Sartoretto, E. R., da Silva, G. S., Schein, A. E., \& Madeira, K. (2019). Contraindicações ao uso de Trombolítico em Pacientes Acometidos por Acidente Vascular Cerebral Isquêmico num Hospital de alta complexidade do sul Catarinense no período de 2012 a 2014. Arquivos Catarinenses de Medicina, 48(1), $108-117$.

Schmidt, M. H., Selau, C. M., da Silva Soares, P., Franchi, E. F., Piber, V. D., \& Quatrin, L. B. (2019). Acidente vascular cerebral e diferentes limitações: uma análise interdisciplinar. Arquivos de Ciências da Saúde da UNIPAR, 23(2).

SEADE. (2021). Portal de estatísticas do Estado de São Paulo. https://www.seade.gov.br/.

SP. (2015). Dia Mundial de Combate ao AVC leva conscientização à Avenida Paulista. Governo do Estado de São Paulo Secretaria de Estado da Saúde. https://www.prefeitura.sp.gov.br/cidade/secretarias/saude/noticias/?p=206029.

SP. (2017). Análise em saúde e vigilância das doenças não transmissíveis. Governo do Estado de São Paulo. http://svs.aids.gov.br/dantps/centrais-deconteudos/paineis-de-monitoramento/mortalidade/gbd-brasil/principais-causas/.

SP. (2019). Hospital Tatuapé completa 50 anos com maior capacidade de atendimento. Governo do Estado de São Paulo. https://www.prefeitura.sp.gov.br/cidade/secretarias/saude/noticias/?p=270429.

SP. (2020). Entenda o que é AVC, como identificar e se proteger. Governo do Estado de São Paulo https://www.prefeitura.sp.gov.br/cidade/secretarias/saude/noticias/?p=306023.

SP. (2021). Número de Estabelecimentos/Serviços próprios da SMS por Coordenadoria Regional de Saúde Município de São Paulo. Governo do Estado de São Paulo. https://www.prefeitura.sp.gov.br/cidade/secretarias/upload/saude/EstabServicos_da_SMS_por_CRS_Marco_2021.pdf=.

Vieira, D. C. D., Callegaro, C. C., Bittencourt, V. L. L., da Silva, T. P., \& Winkelmann, E. R. (2017). Reabilitação de acidente vascular encefálico: revisão de literatura. Revista de Atenção à Saúde (ISSN 2359-4330), 15(52), 89-95. 